\title{
Random Bit Flipping and EXIT Charts for Nonuniform Binary Sources and Joint Source-Channel Turbo Systems
}

\author{
Xavier Jaspar ${ }^{1}$ and Luc Vandendorpe ${ }^{2}$ \\ ${ }^{1}$ Laborelec, Rodestraat 125, B-1630 Linkebeek, Belgium \\ ${ }^{2}$ Communications and Remote Sensing Laboratory, Université catholique de Louvain, Place du Levant 2, \\ B-1348 Louvain-la-Neuve, Belgium
}

Correspondence should be addressed to Xavier Jaspar, xavier.jaspar@uclouvain.be

Received 29 January 2009; Revised 7 June 2009; Accepted 7 August 2009

Recommended by Athanasios Rontogiannis

Joint source-channel turbo techniques have recently been explored a lot in literature as one promising possibility to lower the end-to-end distortion, with fixed length codes, variable length codes, and (quasi) arithmetic codes. Still, many issues remain to be clarified before production use. This short contribution clarifies very concisely several issues that arise with EXIT charts and nonuniform binary sources (a nonuniform binary source can be the result of a nonbinary source followed by a binary source code). We propose two histogram-based methods to estimate the charts and discuss their equivalence. The first one is a mathematical generalization of the original EXIT charts to nonuniform bits. The second one uses a random bit flipping to make the bits virtually uniform and has two interesting advantages: (1) it handles straightforwardly outer codes with an entropy varying with the bit position, and (2) it provides a chart for the inner code that is independent of the outer code.

Copyright (c) 2009 X. Jaspar and L. Vandendorpe. This is an open access article distributed under the Creative Commons Attribution License, which permits unrestricted use, distribution, and reproduction in any medium, provided the original work is properly cited.

\section{Introduction}

Consider the generic system in Figure 1. It involves a serial concatenation at the transmitter and a joint source-channel serial turbo decoder $[1,2]$ at the receiver and is sufficiently general to describe several issues that arise with EXIT charts [3] when the bits $U$ : are not uniform.

Let us consider that the outer component in Figure 1 is a discrete source of symbols followed by a source code that produces a sequence of Nbiased or nonuniform bits $U_{1: N}$ or

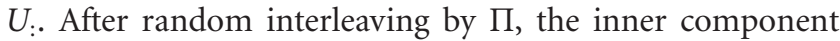
is a channel code that produces a sequence of coded bits $R$ : which are sent across the channel. We assume that the channel code is linear and the channel is binary, symmetric, memoryless, and time invariant. At the receiver, an iterative decoder is used, based on two decoders, one for each code. They exchange log-likelihood ratios (LLRs) iteratively, in a typical joint source-channel serial configuration $[1,2]$. In the following, let $L_{O, \text { : }}^{s}$ be the output LLRs of the (outer) source decoder, let $L_{O \text {,: }}^{c}$ be the deinterleaved output LLRs of the (inner) channel decoder, and let $L_{I,:}^{c}=L_{O,:}^{s}$ and $L_{I,:}^{s}=L_{O \text {,: }}^{c}$ be the corresponding input LLRs.
To assess the convergence of this iterative decoder, the EXIT charts introduced in [3] can be used when the bits are uniform. Unfortunately, when the bits are not uniform, a naive application of the EXIT charts that would neglect the bias might lead to inaccuracy issues. A few contributions already paid attention to some of these issues, notably $[2,4$, 5]. This paper attempts to clarify them all.

This short paper presents concisely two simple histogram-based techniques in Section 2 to estimate the EXIT charts when the bits are not uniform and discusses in Section 3 the (dis)advantages of each. The first technique is based on the system in Figure 1 and is a generalization of [5]; the second one is based on the system in Figure 2 where a random bit flipping is introduced. These techniques are shown to lead to different but equivalent charts under some assumptions. At last, we show that the well-known technique in [6], though historically developed for uniform bits, provides "correct" EXIT charts with nonuniform bits, which is an interesting conclusion for readers familiar with this technique. Please note that, this paper relates only to histogram-based computations of EXIT charts but 


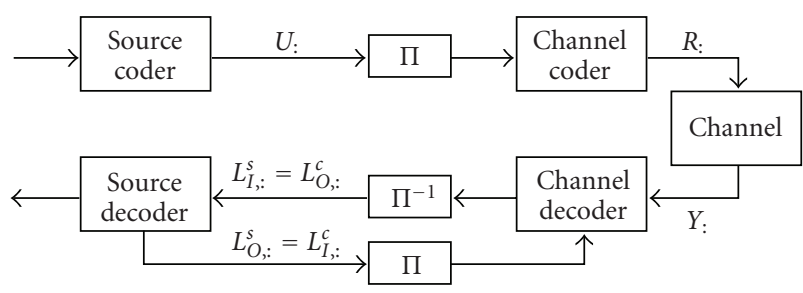

Figure 1: Generic joint source-channel turbo system.

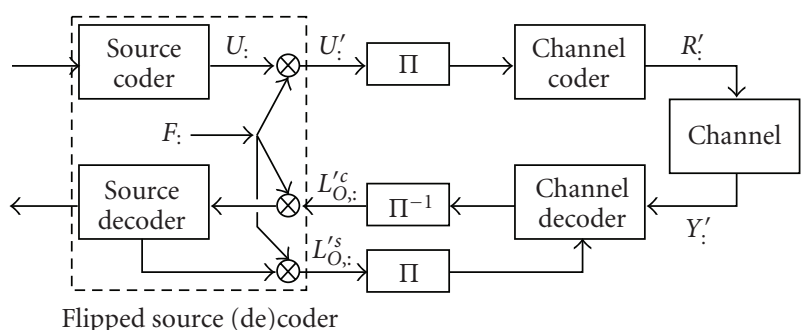

Figure 2: Equivalent system with a random bit flipping, $U_{k}, U_{k}^{\prime}, F_{k} \in\{+1,-1\}$. As in Figure 1, we define $L_{I,:}^{\prime s}=L_{O,:}^{\prime c}$ and $L_{O,:}^{\prime s}=L_{I,:}^{\prime c}$.

the presented ideas and concepts are compatible with the analytical computation proposed in [7].

In the remainder, random variables are written with capital letters and realizations with small letters. $P(z)$ is the abbreviation of the probability $P(Z=z)$. The subsequence $\left(Z_{m}, Z_{m+1}, \ldots, Z_{n}\right)$ is written $Z_{m: n}$ or $Z$ : when $m, n$ can be omitted. $\square\{a\}$ is the indicator function, that is, $\square\{a\}$ equals 1 if $a$ is true, 0 otherwise. $\mathbf{E}\{Z\}$ is the expectation of $Z . I(Y ; Z)$ is the mutual information between $Y$ and $Z$. $H(Z)=I(Z ; Z)$ is the entropy of $Z$. $H_{b}(p)$ is the binary entropy function, that is, $H_{b}(p)=-p \log _{2}(p)-(1-p) \log _{2}(1-p)$.

\section{Computation of the EXIT Charts}

The main results of this section are summarized in Table 1 . For clarity, the first method of computation, which is based on biased bits, is given the name BEXIT while the second one, which is based on flipped bits, is called FEXIT. For the sake of conciseness, some familiarity with [3] is assumed.

2.1. Assumptions, Notations, and Consistency. We assume that the channel code is linear and the channel is binary, symmetric, memoryless, and time invariant. Besides, we assume that the channel and source decoders, taken apart, are optimal. Specifically, let $\mathcal{R}_{k}^{c}=\left(Y_{:}, L_{I, 1: k-1}^{c}, L_{I, k+1: N}^{c}\right)$ and $\mathcal{R}_{k}^{s}=\left(L_{I, 1: k-1}^{s}, L_{I, k+1: N}^{s}\right)$, and assume that the elements in $\mathcal{R}_{k}^{c}$ and in $\mathcal{R}_{k}^{s}$ are independent, then the output LLRs on $U_{k}$ of the channel and source decoders in Figure 1 are considered to be, respectively,

$$
\begin{aligned}
L_{O, k}^{c} & =\log \frac{p\left(\mathcal{R}_{k}^{c} \mid U_{k}=+1\right)}{p\left(\mathcal{R}_{k}^{c} \mid U_{k}=-1\right)}, \\
L_{O, k}^{s} & =\log \frac{p\left(\mathcal{R}_{k}^{s}, U_{k}=+1\right)}{p\left(\mathcal{R}_{k}^{s}, U_{k}=-1\right)} \\
& =\log \frac{p\left(\mathcal{R}_{k}^{s} \mid U_{k}=+1\right)}{p\left(\mathcal{R}_{k}^{s} \mid U_{k}=-1\right)}+L_{U, k},
\end{aligned}
$$

where the source bias $L_{U, k}$ is defined as

$$
L_{U, k} \triangleq \log \frac{P\left(U_{k}=+1\right)}{P\left(U_{k}=-1\right)}, \quad \text { with } 0<P\left(U_{k}=+1\right)<1 .
$$

Note that in the flipped case in Section 2.3, $L_{U, k}^{\prime}=0$. Note also generalizing the results below to $P\left(U_{k}=+1\right) \in\{0,1\}$ (e.g., in the case of pilot bits) is straightforward by taking the limit of $\left|L_{U, k}\right|$ toward infinity where appropriate.

To avoid any confusion, here are some further considerations. Firstly, we prefer not to use the term "extrinsic" for the LLRs $L_{O, k}^{c}$ and $L_{O, k}^{s}$ because this term is used differently by authors in literature. Some authors consider these $L_{O, k}^{c}$ and $L_{O, k}^{s}$ in (1)-(2) as extrinsic; others consider $L_{O, k}^{c}$ (with $Y_{k}$ excluded in the case of a systematic channel code) and $L_{O, k}^{s}-L_{U, k}$ as extrinsic. Secondly, we consider a typical serial concatenation where only the (inner) channel decoder has access to the channel values. It must therefore share this piece of information with the source decoder through $L_{O, k}^{c}$. This is why $L_{O, k}^{c}$ in (1) depends on all channel values $Y$ : through $\mathcal{R}_{k}^{c}$ (even if the channel code is systematic). Similarly, only the (outer) source decoder "knows" the source a priori probabilities. This is why, to share it with the channel decoder, the source bias $L_{U, k}$ is included in $L_{O, k}^{s}$ in (2).

Definition 1 (P-consistency and L-consistency). $L_{p}$ is posterior- consistent or P-consistent with $U$ if

$$
p\left(L_{P}=l, U=+1\right)=e^{l} p\left(L_{P}=l, U=-1\right) .
$$

$L_{L}$ is likelihood-consistent or $L$-consistent with $U$ if

$$
p\left(L_{L}=l \mid U=+1\right)=e^{l} p\left(L_{L}=l \mid U=-1\right) .
$$

Note that if $L_{L}$ is $\mathrm{L}$-consistent, then $L_{L}+L_{U}$ is P-consistent.

Proposition 1. The output LLR of the channel decoder, $L_{O, k}^{c}$ in (1), is L-consistent with $U_{k}$. The output LLR of the source decoder, $L_{O, k}^{s}$ in (2), is P-consistent with $U_{k}$.

Proof. It follows from (1)-(2) and integration of $p\left(\mathcal{R}_{k}^{c} \mid u_{k}\right)$ and $p\left(\mathcal{R}_{k}^{s} \mid u_{k}\right)$.

Note that when the symmetry condition $p(L=-l \mid U=$ $+1)=p(L=l \mid U=-1)$ is satisfied, the consistency in $[4,6]$ and the L-consistency (5) are equivalent. 
TABLE 1: Summary of the two Monte-Carlo methods.

BEXIT charts: with biased bits, Figure 1

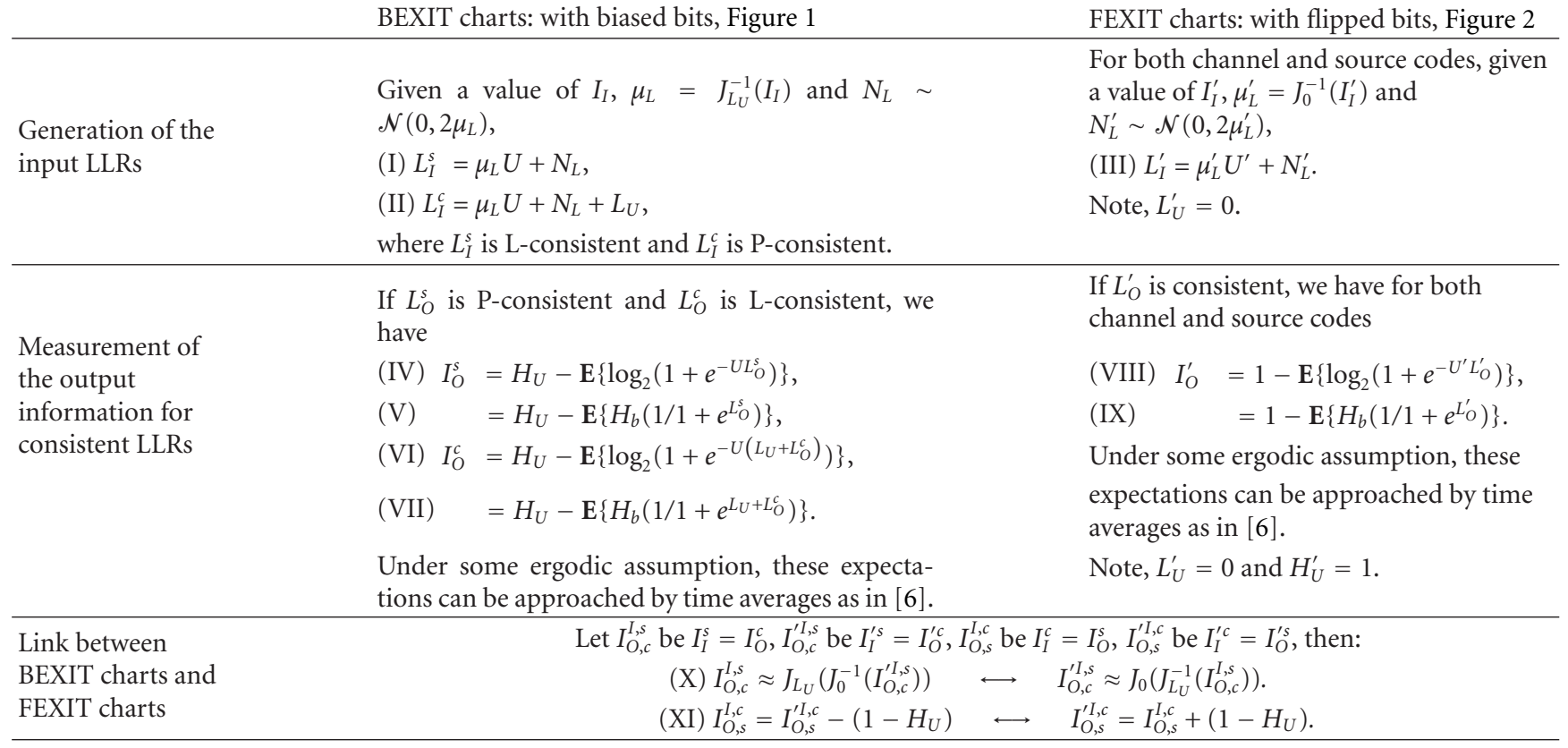

Let us assume that $L_{U}=L_{U, k}$ is independent of $k$; that is, the bits $U_{k}$ have the same entropy $H_{U}=H_{U, k} \triangleq H_{b}\left(P\left(U_{k}=\right.\right.$ $+1)$ ) independently of $k$. Let us then measure the input and output levels of information as

$$
I=\lim _{N \rightarrow+\infty} \frac{1}{N} \sum_{k=1}^{N} I\left(U_{k} ; L_{k}\right) \in\left[0, H_{U}\right],
$$

where $\left(I, L_{k}\right)$ is $\left(I_{I}^{c}, L_{I, k}^{c}\right),\left(I_{I}^{s}, L_{I, k}^{s}\right),\left(I_{O}^{c}, L_{O, k}^{c}\right)$, or $\left(I_{O}^{s}, L_{O, k}^{s}\right)$. As in [3], we will compute the charts $I_{O}^{c}=T^{c}\left(I_{I}^{c}\right)$ and $I_{O}^{s}=T^{s}\left(I_{I}^{s}\right)$ by feeding the decoders with input LLRs and by measuring the level of output information.

Remark 1. The channel (resp., source) decoder will be fed with input LLRs that are independent, P-consistent (resp., Lconsistent, in agreement with Proposition 1), and Gaussian.

Proposition 2. Let $L_{U}^{\star}$ be equal to 0 if $L$ is P-consistent with $U$, and let it be equal to $L_{U}$ if $L$ is $L$-consistent. Then

$$
P(U=u \mid L=l)=\frac{1}{1+e^{-u\left(L_{U}^{\star}+l\right)}} .
$$

Proof. It follows from (4)-(5) and from $P(U=+1 \mid L=$ l) $+P(U=-1 \mid L=l)=1$.

2.2. BEXIT Charts, Figure 1: Biased Bits. We can compute the BEXIT charts of the system in Figure 1 by analytical generalization of the original EXIT charts [3].

(1) Generating the input LLRs. Let us consider a sequence of bits $U$ : generated by the source coder and let us focus on one of these bits, namely, $U$. Given a value $I_{I}=I_{I}^{s}$, the input
LLR $L_{I}^{s}$ on $U$ is generated as in (I) in Table 1 , where $N_{L}$ is a centered Gaussian random variable of variance $2 \mu_{L}$ and the invertible function $J_{L_{U}}(\cdot)$ is given by

$$
\begin{aligned}
J_{L_{U}}(\mu)= & H_{U}-\sum_{u \in\{+1,-1\}} P(U=u) \\
& \cdot \int_{-\infty}^{+\infty} \frac{e^{-(\xi-\mu u)^{2} /(4 \mu)}}{\sqrt{4 \pi \mu}} \log _{2}\left(1+e^{-u\left(L_{U}+\xi\right)}\right) d \xi .
\end{aligned}
$$

This $L_{I}^{s}$ in (I), Table 1, is L-consistent, in agreement with Remark 1.

For the channel decoder, given a value $I_{I}=I_{I}^{c}$, the input LLR $L_{I}^{c}$ on $U$ is generated as in (II), Table 1. Compared to (I), Table 1 , the constant term $L_{U}$ is necessary to make $L_{I}^{c} \mathrm{P}$ consistent.

(2) Measuring the output information. Let us consider the whole sequence of bits $U_{\text {: }}$ and the corresponding output LLRs $L_{O,:}$. For both decoders, we can measure the output information by (6) with

$$
I\left(U_{k} ; L_{O, k}\right)=H_{U}-\mathbf{E}\left\{\log _{2} \frac{1}{P\left(U_{k} \mid L_{O, k}\right)}\right\} .
$$

This expression can be evaluated by approaching $P\left(U_{k}\right.$ । $L_{O, k}$ ) with histogram measurements as in [3].

Assuming consistent LLRs makes things simpler. We can indeed simplify (6) and (9) into (IV) and (VI), Table 1, by 
Proposition 2 and by (9). In addition, we can simplify (IV) into $(\mathrm{V})$ since

$$
\begin{aligned}
\mathbf{E}\left\{\log _{2}\left(1+e^{-U L_{O}^{s}}\right) \mid L_{O}^{s}\right\} & \\
= & \sum_{u \in\{+1,-1\}} P\left(U=u \mid L_{O}^{s}\right) \log _{2}\left(1+e^{-u L_{O}^{s}}\right) \\
& =H_{b}\left(\frac{1}{\left(1+e^{L_{O}^{s}}\right)}\right)=H_{b}\left(\frac{1}{\left(1+e^{-L_{O}^{s}}\right)}\right),
\end{aligned}
$$

where $P\left(U=u \mid L_{O}^{s}\right)$ is given in (7). Similarly, we can simplify (VI) into (VII), Table 1 . Note that (IV), Table 1, is equivalent to $[5$, equation (4)] and is an extension of $[6$, equation (4)].

2.3. FEXIT Charts, Figure 2: Flipped Bits. Let us now consider the system in Figure 2. To make the bit stream uniform, we have introduced a random bit flipping before the interleaver $\Pi$, that is, $U_{k}^{\prime}=U_{k} F_{k}$ for all $k$, where the $F_{k} \in\{+1,-1\}$ are independent and uniformly distributed. At the receiver, the corresponding LLRs are flipped accordingly. By linearity of the channel code and symmetry of the channel, the flipped system in Figure 2 is equivalent to the original system in Figure 1. Consequently, the EXIT charts of the flipped system, namely, FEXIT charts, can be used to characterize the original system. For clarity, all symbols related to the flipped system use a prime $\left({ }^{\prime}\right)$ notation.

With FEXIT charts, we are interested in the exchange of information about $U_{\text {: }}^{\prime}$ between the channel decoder and the flipped source decoder in Figure 2. Since the bits $U_{k}^{\prime}$ are uniform, we can use the results obtained so far with $L_{U}^{\prime}=0$ and $H_{U}^{\prime}=1$ (see Table 1). This is equivalent to [3]; in particular, the function $J_{0}(\cdot)=J_{L_{U}=0}(\cdot)$ is related to the function $J(\cdot)$ in [3] with $J_{0}\left(\mu^{\prime}\right)=J\left(\sqrt{2 \mu^{\prime}}\right)$.

\section{Transformations, Equivalence, and Discussion}

3.1. Transformations and Equivalence. The BEXIT and FEXIT charts are equivalent under the assumptions of Section 2.1, up to the approximation (X), Table 1. Indeed, under these assumptions, transformations to obtain the FEXIT chart from the BEXIT chart, and vice versa, are given in (X) and (XI) and Table 1. To prove (X) and Table 1, let us consider $L_{I}^{s}$ given in (I), Table 1 , in the biased case. If we apply the flipping $F$ on $L_{I}^{s}$, we get $L_{I}^{\prime s}=L_{I}^{s} F=\mu_{L} U F+N_{L} F=$ $\mu_{L} U^{\prime}+N_{L} F$, and it is self-evident that this $L_{I}^{\prime s}$ is equivalent to (III), Table 1 , if $\mu_{L}=\mu_{L}^{\prime}$, that is, if $J_{L_{U}}^{-1}\left(I_{I}^{s}\right)=J_{0}^{-1}\left(I_{I}^{\prime s}\right)$, which proves (XI), Table 1, for consistent Gaussian LLRs. For consistent non-Gaussian LLRs, we can invoke the empirical robustness of EXIT charts with respect to the statistical model of the LLRs and assume that (X), Table 1, is a sufficient approximation, hence the approximation symbol “ $\approx$ ”. To prove (XI), Table 1, we use the following equalities:

$$
\begin{aligned}
I_{O}^{s}-H_{U} & =-\mathbf{E}\left\{\log _{2}\left(1+e^{-U L_{O}^{s}}\right)\right\}, & & \text { by }(\mathrm{IV}), \\
& =-\mathbf{E}\left\{\log _{2}\left(1+e^{-U^{\prime} L_{O}^{\prime s}}\right)\right\}, & & \\
& =I_{O}^{\prime s}-1, & & \text { by (VIII) }
\end{aligned}
$$

where (12) comes from $U^{\prime} L_{O}^{s}=(U F)\left(L_{O}^{s} F\right)=U L_{O}^{s}$.

3.2. Simulation Results. To illustrate the equivalence, let us compute the charts of the following (nonoptimized) system. The outer component in Figure 1 is a memoryless source of 3 symbols with probabilities $0.85,0.14$, and 0.01 , transcoded, respectively, by the variable length codewords $(+1),(-1,-1)$, $(-1,+1,-1)$, leading to $P(U=+1)=0.741$ and $H_{U}=$ 0.825 . The channel code is a rate $-(1 / 2)$ recursive systematic convolutional code with forward generator $35_{8}$ (in octal) and feedback generator $23_{8}$. The channel is an additive white Gaussian noise channel with binary phase-shift keying and $E_{b} / N_{0}=1.4 \mathrm{~dB}-E_{b}$ is the energy per bit of entropy and $N_{0} / 2$ the double-sided noise spectral density. Note that the source decoder is based on the BCJR algorithm [8] on Balakirsky's bit-trellis [9].

The BEXIT and FEXIT charts of the system are given in Figures 3 and 4 . The solid lines show the charts obtained with the methods described in Sections 2.2 and 2.3. The data points show the BEXIT and FEXIT charts obtained by applying the transformations (X)-(XI), Table 1, respectively, on the FEXIT and BEXIT charts given in solid lines. The good match between the data points and the solid lines illustrates the equivalence between FEXIT and BEXIT charts. All system configurations we have tested confirm this equivalence, at least in the tested range $P(U=+1) \in[0.05,0.95]$.

Finally, when we neglect the bias and apply blindly the original method of [3]—generating the input LLRs with [3, equation (9)] and [3, equation (18)] and measuring the output information with [3, equation (19)]-, we obtain actually the FEXIT chart of the channel decoder for the channel decoder and the dashed line in Figure 4 for the source decoder. As we can see, these charts intersect each other and give a prediction of convergence that is too pessimistic.

3.3. Discussion. In terms of (dis)advantages of each method, FEXIT charts, unlike BEXIT charts, are limited to linear channel codes and symmetric channels. Nevertheless, when the channel code is linear and the channel symmetric, FEXIT charts have at least two benefits. Firstly, the FEXIT chart of the (inner) channel code is independent of the (outer) source code; so we do not need to recompute it when the source code changes. By contrast, we need to recompute the BEXIT chart of the channel code when $L_{U}$ changes since it depends on $L_{U}$ (see (II), (VI), and (VII) in Table 1). Secondly, FEXIT charts can handle very easily (outer) source codes with an entropy $H_{U, k}$ that depends on $k$-recall that we have assumed that $L_{U, k}$, therefore $H_{U, k}$, is independent of $k$ in Section 2.1-, simply because the random bit flipping makes the entropy 


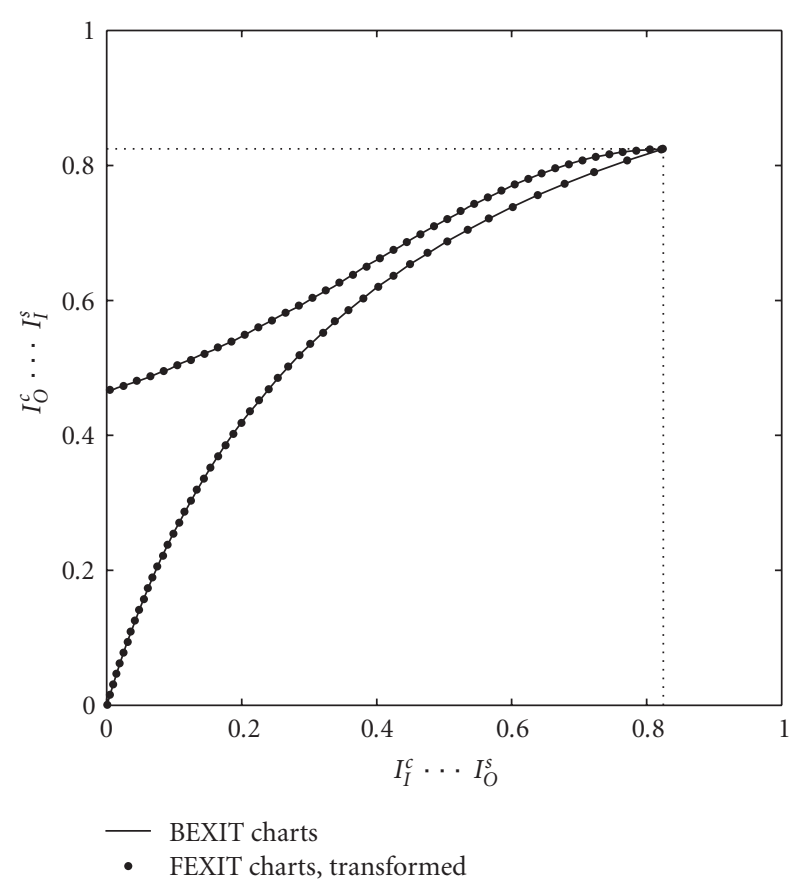

FIgURE 3: BEXIT charts of the system described in Section 3.1.

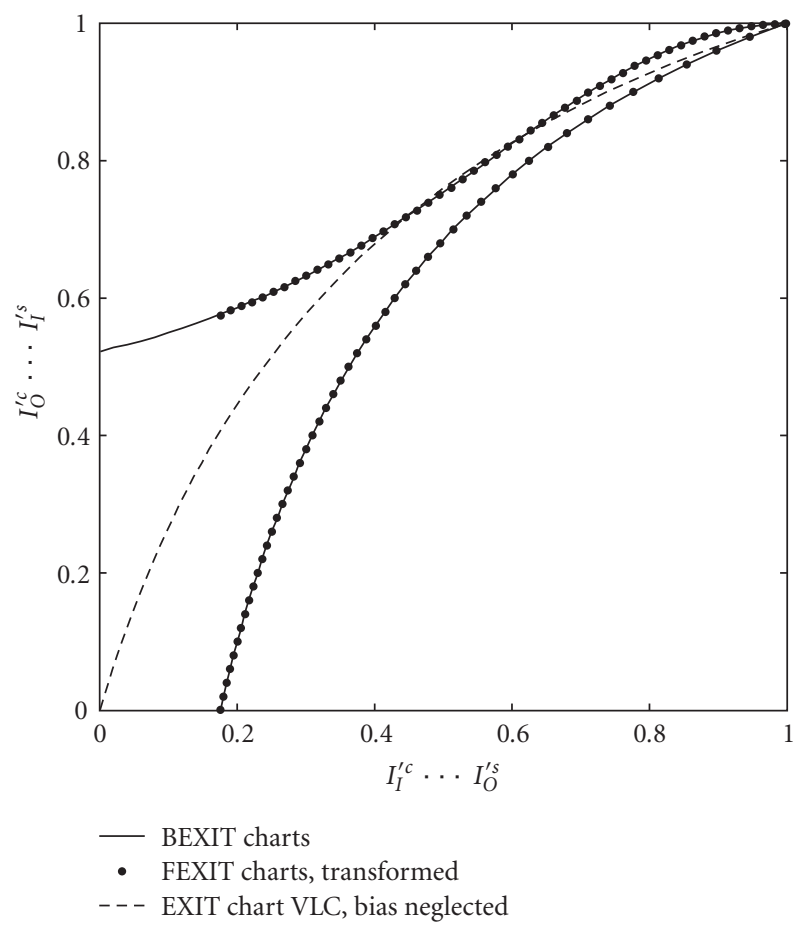

FIGURE 4: FEXIT charts of the system described in Section 3.1.

equal to 1 for all $k$. On the contrary, no method to handle them with BEXIT charts is known to the authors. Note that a varying $H_{U, k}$ is not uncommon in practice: fixed length codes, variable length codes with periodic bit-clock trellises, mixture of such codes, and so forth.
At last, among related contributions in literature, the well-known technique in [6] is of particular interest. Though historically developed for uniform bits, this technique gives without bit flipping a correct prediction of convergence when the channel code is linear and the channel is symmetric. It computes indeed [6, equation (5)] the output information as (with some mathematical rewriting)

$$
I_{O}^{[6]}=1-\mathbf{E}\left\{\log _{2}\left(1+e^{-U L}\right)\right\} .
$$

Since $U^{\prime} L^{\prime}=(U F)(L F)=U L$, it is equivalent to (VIII), Table 1, and thus to the FEXIT charts presented in this paper.

\section{Conclusion}

Two methods have been presented to handle nonuniform bits in the computation of EXIT charts. Though proved to be equivalent for the prediction of convergence under certain assumptions, they have different pros and cons. For example, the FEXIT method is restricted to linear inner codes and symmetric channels while the BEXIT method is not. But the FEXIT method handles very easily a mixture of bits having different entropies and offers a chart for the inner channel decoder (of a serial concatenation) that is independent of the outer source code, unlike the BEXIT method, which simplifies greatly subsequent optimizations of the concatenated code. In practice, both methods are therefore complementary and help to analyze joint sourcechannel turbo systems via EXIT charts.

\section{Acknowledgment}

The authors greatly thank the reviewers for their constructive comments. The work of X. Jaspar is supported by the F.R.S.FNRS, Belgium.

\section{References}

[1] A. Guyader, E. Fabre, C. Guillemot, and M. Robert, "Joint source-channel turbo decoding of entropy-coded sources," IEEE Journal on Selected Areas in Communications, vol. 19, no. 9, pp. 1680-1696, 2001.

[2] M. Adrat and P. Vary, "Iterative source-channel decoding: Improved system design using EXIT charts," EURASIP Journal on Applied Signal Processing, vol. 2005, no. 6, pp. 928-947, 2005.

[3] S. ten Brink, "Convergence behavior of iteratively decoded parallel concatenated codes," IEEE Transactions on Communications, vol. 49, no. 10, pp. 1727-1737, 2001.

[4] J. Hagenauer, "The EXIT chart-introduction to extrinsic information transfer in iterative processing," in Proceedings of the 12th European Signal Processing Conference (EUSIPCO '04), Vienna, Austria, September 2004.

[5] N. Dütsch, "Code optimisation for lossless compression of binary memoryless sources based on FEC codes," European Transactions on Telecommunications, vol. 17, no. 2, pp. 219-229, 2006.

[6] M. Tüchler and J. Hagenauer, "EXIT charts of irregular codes," in Proceedings of the 36 th Annual Conference on Information Sciences and Systems (CISS '02), Princeton, NJ, USA, March 2002. 
[7] M. Adrat, J. Brauers, T. Clevorn, and P. Vary, "The EXITcharacteristic of softbit-source decoders," IEEE Communications Letters, vol. 9, no. 6, pp. 540-542, 2005.

[8] L. R. Bahl, J. Cocke, F. Jelinek, and J. Raviv, "Optimal decoding of linear codes for minimizing symbol error rate," IEEE Transactions on Information Theory, vol. 20, no. 2, pp. 284-287, 1974.

[9] V. B. Balakirsky, "Joint source-channel coding with variable length codes," in Proceedings of the IEEE International Symposium on Information Theory (ISIT '97), p. 419, Ulm, Germany, July 1997. 